\title{
Altering a computerized laboratory test order form rationalizes ordering of laboratory tests in primary care physicians
}

\section{Seppänen, Katri}

2016

Seppänen , K, Kauppila , T I , Pitkälä , K H , Kautiainen , H , Puustinen , R , livanainen , A \& Mäki , T 2016, ' Altering a computerized laboratory test order form rationalizes ordering of laboratory tests in primary care physicians ' , International Journal of Medical Informatics, vol. 86 , pp. 49-53 . https://doi.org/10.1016/j.ijmedinf.2015.11.013

http://hdl.handle.net/10138/223828

https://doi.org/10.1016/j.jmedinf.2015.11.013

publishedVersion

Downloaded from Helda, University of Helsinki institutional repository.

This is an electronic reprint of the original article.

This reprint may differ from the original in pagination and typographic detail.

Please cite the original version. 


\title{
Altering a computerized laboratory test order form rationalizes ordering of laboratory tests in primary care physicians
}

\author{
Katri Seppänen ${ }^{a}$, Timo Kauppila ${ }^{\text {b,* }}$, Kaisu Pitkälä ${ }^{b}$, Hannu Kautiainen ${ }^{b}$, Raija Puustinen ${ }^{c}$, \\ Antti Iivanainen ${ }^{c}$, Tiina Mäki ${ }^{\mathrm{a}}$ \\ a Department of Primary Health Care Laboratory Services, Helsinki University Central Hospital, Laboratory Services (HUSLAB), Paciuksenkatu 29, P.O. Box \\ 720, 00027 HUS Helsinki, Finland \\ ${ }^{\mathrm{b}}$ Department of General Practice and Primary Healthcare, University of Helsinki, and Unit of Primary Health Care, Helsinki University Central Hospital, \\ (HUS), Tukholmankatu 8 B, P.O. Box 20, 00014 Helsinki, Finland \\ ${ }^{\mathrm{c}}$ Health Center of City of Helsinki, Siltasaarenkatu 13, P.O. Box 1, 00099 City of Helsinki, Finland
}

\section{A R T I C L E I N F O}

\section{Article history:}

Received 22 March 2015

Received in revised form

25 November 2015

Accepted 26 November 2015

\section{Keywords:}

Laboratory test

Computerized laboratory test order form

Alanine transaminase

Aspartate transaminase

C-reactive protein

Erythrocyte sedimentation rate

\begin{abstract}
A B S T R A C T
Background: To reduce physicians' inappropriate laboratory requests for their patients, administrators have used methods such as modifying a laboratory request order form with an agreed requesting protocol for the most common diagnoses in primary health care.

Objective: To study the effects of removing the erythrocyte sedimentation rate (ESR) and aspartate transaminase (AST) which are considered of limited clinical value for primary care clinical decisionmaking from a computerized laboratory test order form. These tests were removed to another new view from the electronic laboratory menu where the physicians, instead of just ticking the desired test from the list, had to do 4-8 s extra work by writing down the abbreviation to order the test.

Methods: An observational controlled prospective study based on a before-after design was performed by removing AST and ES from the laboratory test order form of the computerized laboratory system for all primary care in the city of Helsinki, Finland. The numbers of annual and monthly use of AST and ESR and their controls, alanine transaminase (ALT) and C-reactive protein (CRP) ordered by General practitioners (GPs) was recorded over an eight-year period: four years before and a four years after the removal of AST and ES.

Results: Removing AST and ESR from the computerized laboratory test order form decreased their use by up to $90 \%$, whereas the use of the control tests increased throughout the follow-up period. The variation in use of these removed tests also decreased.

Conclusion: Removing a laboratory test from a computerized laboratory test order form may significantly reduce GPs' use of the laboratory test. Further studies are needed, however, to ensure the safety of this type of intervention.
\end{abstract}

(c) 2015 Elsevier Ireland Ltd. All rights reserved.

\section{Introduction}

Ample resources have been invested in efforts to improve the quality of health care and its cost-effectiveness [1]. These actions also aim to improve care from the patients' perspective. Due to limited resources, decision makers have a responsibility to decide how to use these resources to maximize the benefits [1].

\footnotetext{
* Corresponding author. Fax: +358919127536.

E-mail addresses: katri.seppanen@hus.fi (K. Seppänen),timo.kauppila@fimnet.fi (T. Kauppila),kaisu.pitkala@helsinki.fi (K. Pitkälä), hannu.kautiainen@medcare.fi (H. Kautiainen), raija.puustinen@helsinki.fi (R. Puustinen), antti.iivanainen@helsinki.fi (A. Iivanainen), tiina.maki@hus.fi (T. Mäki).
}

Inadequate use of laboratory tests in primary care has been a target of such interventions [2-6]. To reduce physicians' inappropriate laboratory requests for their patients, administrators have used methods such as involving a laboratory request menu with an agreed requesting protocol for the most common diagnoses in primary health care [2]. When incorporated into an electronic laboratory request menu, this system provides electronic reminders, which have proved effective in improving the quality of care [7-9]. These reminders have been reported to enhance better prescribing practices [10], better control over the treated disease [11] and further better recording in patient charts [12].

Former findings suggested that laboratory test form design influences test ordering by general practitioners (GPs) [13]. This led to some relatively short experiments in primary care system 
of Israel $[14,15]$. Plain manipulation of computerized laboratory test order forms of primary care practitioners by deleting certain tests was found to decrease use of these deleted laboratory tests by $31-41 \%$ relative to the pre-intervention month, with a further decrease by $36-53 \%$ the following month in a short two month experiment [14]. In a more extensive three year work, tests that were removed from a computerized laboratory order form showed an decrease of $27 \%$ in the first year and a further $19,2 \%$ decrease in the following year [15]. No educational programmes were reported to be included in these interventions $[14,15]$.

In 2006, the laboratory menu in Helsinki primary care was a modification of a previously described laboratory menu [2] that was incorporated into an electronic laboratory system and patient medical records. The menu offered the most commonly used laboratory tests in primary care and reminded the GPs of the availability and usability of these tests. Less frequently used tests had to be ordered with abbreviations available from the computerized laboratory handbook, similar as described by Shalev [15]. So, this computerized laboratory test order form directed GPs' use of laboratory tests in primary care towards more appropriate use. The form contained 69 of the most commonly used laboratory tests, yet allowed clinicians to combine tests on the menu in any manner he or she desired, even if the combinations were inappropriate for clinical decision-making.

Despite continuous medical training on the appropriate use of lab tests GPs may continue to order such lab test combinations that have limited value in clinical decision-making [2]. Although several putative reasons for this behavior - often related to what is known as so-called "defensive medicine" performed by primary care doctors - have been suggested [4] the basic cause for this behavior remains unclear. However, we also hypothesized that easy access to laboratory tests in the computerized laboratory test order form and the public funding of medical care [16] with no sanctions, may also have contributed to the physicians' behavior.

To intervene in this problem, the electronic laboratory menu was modified. Certain tests which were scientifically [3-5] considered to be of limited clinical value for primary care clinical decisionmaking were removed from the computerized laboratory test order form regardless of how often the clinicians used to prescribe these tests. The medical education alone has not always been reported to be enough to produce significant changes in physicians' behavior in laboratory test prescribing [3]. This was also our experience before the present intervention because neither team teaching nor feed-back delivered in superior-subordinate or development discussions altered clearly laboratory test prescribing practices in the local public primary care before the present intervention. Therefore, we also wanted to study what happens to the use of those tests that were removed from our computerized laboratory test order form after the intervention. In this study we explored the effects of deleting erythrocyte sedimentation rate (ESR) and aspartate transaminase (AST) from this laboratory menu by comparing GPs' usage of them during the 4-year period before with 4-year period after the removal.

\section{Methods}

\subsection{Design}

The present experiment is a prospective study with a beforeand after-design in the primary care of the capital of Finland.

\subsection{Setting}

The study was performed in the primary care center for the City of Helsinki and in the HUSLAB, a municipal commercial enter- prise providing laboratory services for the City of Helsinki and 21 other communities. The Helsinki primary care center serves about 550,000 inhabitants of the city.

\subsection{Participants}

The subjects of our study are GPs ( $n=272)$ who serve this population in primary care center. All the data was gathered and handled in such a way as to maintain patient and doctor anonymity. The registry keeper (health authorities of Helsinki and HUSLAB) accorded permission for the study (permission number HEL 2012-01029T 13 02 01).

\subsection{Procedure and measurement}

In February-March 2007 two widely used laboratory tests considered to have low clinical relevance, ESR and AST [3-5], which were relatively widely used in Helsinki primary care, were removed from the computerized laboratory test order forms. This intervention was implemented with a change in the computer system resulting in a new form excluding these two tests. This intervention was supported with short meetings for delivering information (maximum $1 \mathrm{~h}$ ) in order to explain to GPs the reasons for removing these tests from the menu. Thus, the GPs were still able to order the removed tests but they had to find their abbreviations or code numbers from the electronic laboratory book attached to the system or to remember these codes to order them. To order the intervened laboratory tests, a GP had to do 4-8 s extra work because, similarly to Shalevs' work [15], he/she had to open an another new view from the electronic laboratory menu and write down the abbreviation of ESR or AST on an appropriate field of that view. Alanine transaminase-test (ALT) which served as a control to AST-test and C-reactive protein-test (CRP) which served as a control to ESR-test were not removed from the computerized laboratory test order form. To order these control tests, a GP just had to to select the required laboratory test on the menu.

The ESR test is a highly non-specific test for inflammation and tissue damage, and a pathological ESR test result alone leads to clinical actions only when it is highly abnormal $(>50 \mathrm{~mm} / \mathrm{h})$, unlike the CRP test which is quick and clinically relevant to decision-making, especially in acute situations and severe clinical conditions [17]. The AST test does not increase the clinical information obtained with the ALT-test, yet GPs in Helsinki primary care generally used these two tests together.

The annual frequencies of the ordered tests (ALT, AST, ESR and CRP) served as the outcome measures. We were also able to compare monthly variations of the ALT and AST tests, which were often used simultaneously prior to the intervention. The follow-up prior to the intervention began in January 2003 and ended in December 2006, whereas the after intervention follow-up began in February 2007 and lasted through December 2010.

\subsection{Statistical analysis}

The numbers of annual laboratory test (per 1000 blood samplings) with $95 \%$ confidence intervals (CI) were calculated assuming a Poisson distribution. Rate ratios (RR) and statistical significance between observation years were calculated by using Poisson regression models or negative binomial regression models, as appropriate. The assumptions of overdispersion in the Poisson model were tested with the Lagrange multiplier test. The total number of laboratory samples prescribed in the health care of the City of Helsinki was used in analysis. The number of blood samples varied between 516,261 samples/year (minimum, in 2005) and 614,397 (maximum, in 2004) and the number of visits to GPs varied between 441,028 visits/year (minimum, in 2009) and 484,304 (maximum, in 
Use of ALT and AST tests before and after removing the AST test (dashed line) from the laboratory menu

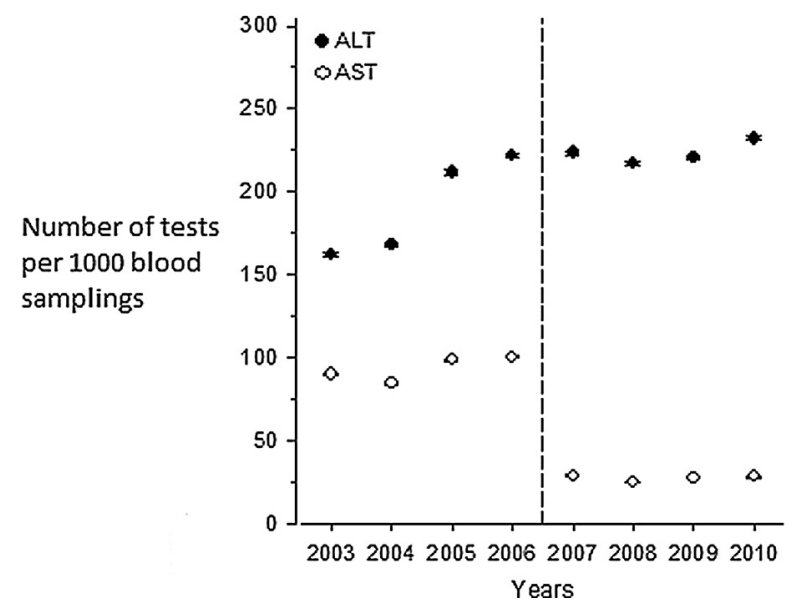

Fig. 1. Use of ALT and AST tests before and after removing the AST test from the laboratory menu. Means and 95\% confidence levels are presented.

2005). Stata 13.1, StataCorp LP (College Station, TX, USA) statistical package was used for the analyses.

\section{Results}

The frequency for prescribing the ALT test increased from 162.1 (CI 95\%: 161.1-163.2) to 232.1 tests/1000 blood samples (CI 95\%: 230.9-233.3) during the follow-up [RR=1.43 (95\% CI: $1.41-1.44)$, $p<0.001]$. Before the intervention, the frequency of prescribing the AST test increased slightly during the period between 2003 (89.97 tests/1000 blood samples, CI 95\%: 89.2-90.7) and 2006 (100.6 tests/1000 blood samples, CI 95\%: 99.7-101.4) [RR=1.12 (95\% CI: 1.10-1.13), $p<0.001$ ], but after the intervention, the frequency eventually decreased to 28.5 tests/1000 blood samples (CI 95\%: $28.0-28.9$ ) in 2010 [RR=0.32 (95\% CI: 0.31-0.33), $p<0.001]$ (Fig. 1).

Similarly, the frequency of prescribing the CRP test increased from 273.0 tests/1000 blood samples (CI 95\%; 271.7-274.3) to 344.7 tests/1000 blood samples (CI 95\%: 343.2-346.2) during the sevenyear follow-up [RR $=1.26$ (95\% CI: 1.25-1.27), $p<0.001$ ]. Before the intervention, the frequency of prescribing the ESR test remained relatively stable between 2003 (139.4 tests/1000 blood samples, CI 95\%: 138.5-140.4) and 2006 (137.6 tests/1000 blood samples, CI 95\%: 136.6-138.6) [ RR $=0.99$ (95\% CI: 0.98-1.01), $p=0.055]$. The intervention was associated with a decrease. In 2010, the frequency of ESR tests was 60.9/1000 blood samples (CI 95\%: 60.2-61.5) [RR=0.44 (95\% CI: 0.42-0.45), $p<0.001$ ] (Fig. 2).

The monthly variation in the use of AST tests disappeared after the intervention, whereas that same variation remained in the use of the ALT test (Fig. 3). The lowest frequencies in the use of the laboratory tests studied occurred in July, which is the most common month for annual leave in Finland.

\section{Discussion}

In this study, the removal of AST and ESR from the computerized laboratory test order form led to GPs significantly lower use of these laboratory tests. The former interventions (e.g. team teaching and feed-back delivered in superior-subordinate or development discussions) alone altered only modestly if at all laboratory prescribing practices towards better use of guidelines. However, modifying electronic laboratory test order form supported clearly
Use of CRP and ESR tests before and after removing the ESR test (dashed line) from the laboratory menu

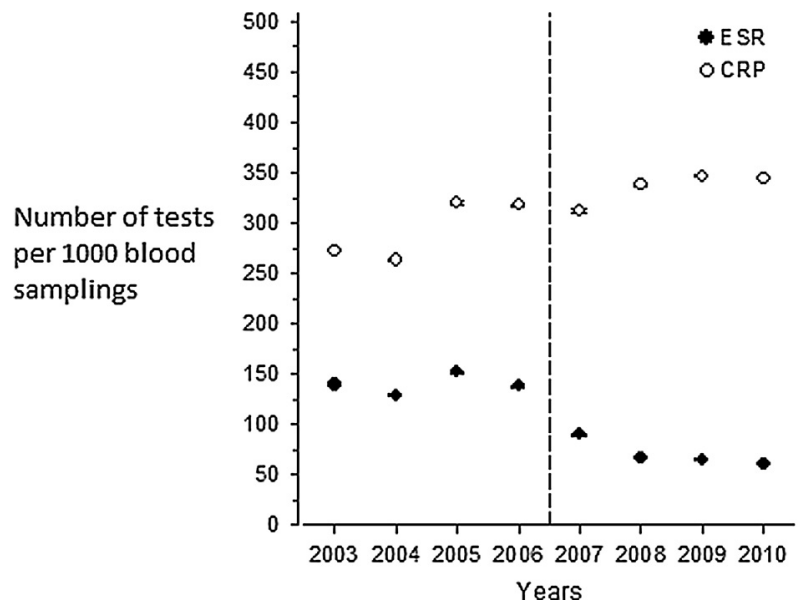

Fig. 2. Use of CRP and ESR tests before and after removing the ESR test from the laboratory menu. Means and 95\% confidence levels are presented.

\section{Development of the monthly use of ALT and AST tests before and after the removing AST test (solid line) from the laboratory menu}

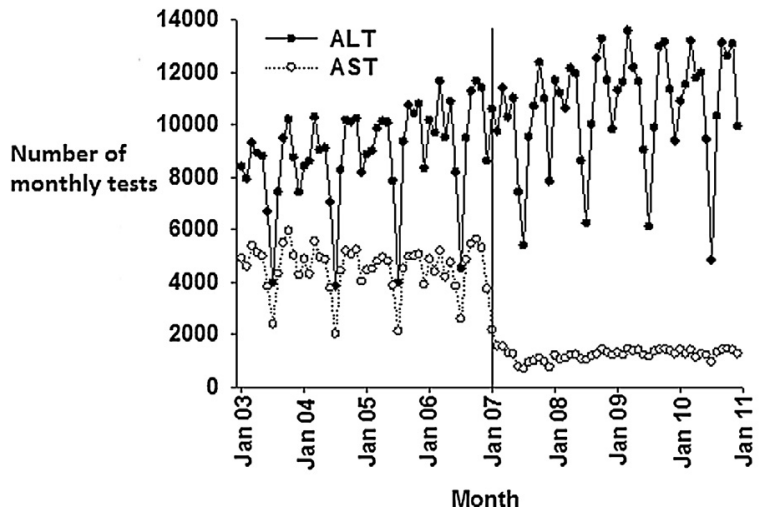

Fig. 3. Monthly use of ALT and AST tests before and after removing the AST test from the laboratory menu.

use of guideline-based laboratory prescribing. The effect of this intervention was stronger than described in the former similar studies $[14,15]$ and highly sustainable. The results suggest that in efficacy of promoting desirable changes the present type of intervention was among the most efficient ones when compared with any other reported effects of on-screen, point of care computer reminders on processes and outcomes of care [6-8].

Medical education alone has not always been reported to be enough to produce the change in physicians' behavior just in laboratory test prescribing in all studies [2,3]. In absence of a control group, we cannot claim that the education we provided before intervention had no effect on laboratory test prescribing in the present experiment. However, this putative effect of education was small. Feed-back delivered in superior-subordinate or development discussions were not either very effective in altering laboratory test prescribing practices in the local public primary care during our four year control period before the present intervention. In Sweden, it was reported that education alone was enough to reduce inappropriate laboratory test use of GPs [5]. For unknown reason, educating GPs was just not, however, enough to change significantly clinical practices in this particular matter with the present GP population. Therefore, something else was worth try- 
ing to enhance the effects of the education which we were able to offer before the intervention and to make GPs follow guidelines in laboratory prescribing.

As an alternative strategy for the present intervention, electronic reminders which have usually been thought to improve the quality of work [6-9] might be applied. Although electronic reminders are effective and useful, GPs ' compliance to use them is not necessary always high for various reasons [18]. Thereby, other methods may also enhance the use of modified laboratory test order forms or electronic reminders as a tool for improving the quality of care when applied simultaneously. Different educational interventions [19-22] could serve as an adjunctive method for these interventions to improve the quality of laboratory prescribing. In the present experiment, the GPs received minimal information in short meetings explaining why these tests were removed from the computerized laboratory test order form, but no training on how to use the available test selection. Thus, the most effective component in the present intervention was not necessarily increasing knowledge or smoothing usability, but rather "selling inconvenience" by producing extra work. This inconvenience was not a major one, since all the tests removed from the computerized laboratory test order form were still relatively easily available to the GPs after the intervention producing only a few seconds extra work as in former studies [14,15]. Yet, defensive behaviour, fear of uncertainty, lack of experience, use of protocols and guidelines, "routine" clinical practice, inadequate educational feedback, clinician's unawareness of the cost of tests, belief that test results are considered more significant than physical examination or history taking, desire to be complete, and collective ordering have been reported to be putative reasons identified for excessive ordering of tests by physicians [23-25]. Nevertheless, the present intervention was highly effective against these factors since it successfully decreased the use of the AST test by as much as $80-90 \%$. A putative explanation for the present observation is that others than professional considerations, e.g. context-related factors, modulate strongly the use of laboratory tests in primary care [26].

The present intervention originated from the administration of primary care and was performed by modulating the information technology (IT) used. Relatively little prior research is available about the efficacy of modulating IT as a method for improving the quality of care and this literature is partially inconsistent [27]. The present intervention was planned by the clinical leaders of the local primary care and this clinical leadership is essential in developing and performing IT-based interventions [28]. Because clinical leaders belong to the health care staff, too, the present finding is in line with the evidence suggesting that projects originating from the patient or health care staff initiative and aimed at improving the quality of care are more likely to be successfully implemented than those originating from the administration of health care [29]. Yet, the present system offers one possibility for administration to drive IT-based intervention to modulate behavior of clinicians, in this case GPs. Since any single implementation alone is unlikely effectively instill good clinical practice [7] other interventions suitable for enhancing the presently described intervention should be considered. Training programs [19-21] and auditing with feedback programs [22] could enhance the present intervention by explaining appropriately to the staff why the intervention was performed and how to use the laboratory tests adequately.

It must be noted here that modulating laboratory test order form is not the same as computerized support for clinical decisionmaking. Decision support technologies purport to optimize the use of diagnostic tests in clinical practice by offering direct help with diagnostics and treatment through actively giving advice on how to proceed [30]. Computerized support of clinical decision-making also serves to inhibit the underuse and overuse of diagnostic tests and has important implications for health outcomes and costs $[8,9,31]$.

The present data suggest that laboratory test order forms may also tempt physicians to overuse the requests, if made too easy. Thus, physicians may not always consider the consequences of their actions. Therefore, administration should always very critically consider which electronic forms for different kind of prescriptions to take into practice and which not.

Present intervention offers one possibility for the administration to drive IT-based intervention to modulate the clinical behavior of physicians which may hypothetically lead to considerable cost savings. Controlling costs is one of the factors which brought overutilization of laboratory tests to the interests of the administration in primary care [2-5]. Yet, further work with a larger scale of intervention should focus on rapid access menus before studying the effect of the present intervention on overall laboratory costs.

\subsection{Limitations}

Before-after setting was not optimal to study the present intervention. An optimal situation would have been to be able to arrange a control group for the present intervention. Unfortunately, it was not possible to arrange that in the primary care of Helsinki.

The main question that remains unanswered is whether the present guidance of clinical activities by "selling inconvenience" (in this case 4-8s extra work) is safe. In the present case which eradicates the double-use of AST-ALT tests, should see no major problems because the both tests are usually requested as screening methods for hepatocellular injury, and for that purpose, ALT is more specific and sensitive [17]. The situation is a bit different when comparing CRP and ESR tests because they are clinically used in slightly different situations: ESR is a rather unspecific and slowly reacting indicator of inflammation and used mainly to detect the activity of certain chronic inflammations such as rheumatoid arthritis. CRP, on the other hand, serves merely to detect the activity of more acute inflammations/infections [17]. Nevertheless, further studies of the safety of this intervention are underway.

\section{Conclusions}

Modulating a computerized laboratory test order form reduced use of inappropriate laboratory tests by $80-90 \%$ and led to GPs more thoroughly considering the use of laboratory tests.

\section{Authors' contribution}

KS wrote the manuscript, ideated the collection of the data and performed it, and performed the intervention. TK wrote the manuscript and organized the study group, designed the study. $\mathrm{KP}$ collected the research team, ideated the study and wrote the manuscript. HK performed the statistical analysis. RP planned and performed the intervention. AI gave the original idea to study this intervention, planned and performed the intervention. TM wrote the manuscript, ideated the collection of the data and planned and performed the intervention. All authors revised the final version of the manuscript and approved it and are thereby accountable for all aspects of the work in ensuring that questions related to the accuracy or integrity of any art of the work are appropriately investigated and resolved.

\section{Conflict of interests}

The authors declare no conflict of interests. 


\section{Summary table}

What was already known on the topic:

- Due to limited health care resources, decision makers have a responsibility to decide how to intervene in health care system to maximize the benefits and thereby inadequate use of laboratory tests in primary care has become a target of such interventions.

- Former findings suggested that laboratory test form design influences test ordering.

- Plain manipulating computerized laboratory test order forms of primary care practitioners by making it somewhat more inconvenient to order clinically less relevant laboratory tests via removing these articles from the test order forms was found to decrease the use of these tests.

What this study added to our knowledge:

- When applicable, manipulating static order forms, in this case computerized laboratory test order form, of primary care practitioners offers one possibility for the administration to drive IT-based intervention to modulate the clinical behavior of GPs in a highly sustainable manner.

- The present intervention had high efficacy in promoting desirable changes when compared with any other reported effects of on-screen, point of care computer reminders on processes and outcomes of care.

- Administration should always very carefully consider which static electronic order forms to take into practice and which not.

\section{Acknowledgements}

The authors thank Stephen Stalter for his valuable comments on language. This study was supported by the City of Helsinki.

\section{References}

[1] J. Grimshaw, M. Eccles, R. Thomas, et al., Toward evidence-based quality improvement. Evidence (and its limitations) of the effectiveness of guideline dissemination and implementation strategies 1966-1998, J. Gen. Intern. Med. 21 (Suppl. 2) (2006) S14-S20.

[2] J.M. Castellví-Boada, X. Castells-Oliveres, Appropriateness of physicians' requests of laboratory examinations in primary health care: an over- and under-ulilisation study, Clin. Chem. Lab. Med. 37 (1999) 65-69.

[3] A. Larsson, S. Biom, M.L. Wernroth, et al., Effects of an education programme to change clinical laboratory testing habits in primary care, Scand. J. Prim. Health Care 17 (1999) 238-243.

[4] W.H. Verstappen, T. van der Weijden, J. Sijbrandij, et al., Effect of a practice-based strategy on test ordering performance of primary care physicians: a randomized trial, JAMA 289 (2003) 2407-2412.

[5] M. Mindemark, A. Larsson, Long-term effects of an education programme on the optimal use of clinical chemistry testing in primary health care, Scand. J. Clin. Lab. Invest. 69 (2009) 481-486.

[6] M.A. van Wijk, J. van der Lei, M. Mosseveld, et al., Assessment of decision support for blood test ordering in primary care. a randomized trial, Ann. Intern. Med. 134 (2001) 274-281.

[7] M. Prior, M. Guerin, K. Grimmer-Somers, The effectiveness of clinical guideline implementation strategies-a synthesis of systematic review findings, J. Eval. Clin. Pract. 14 (2008) 888-897.
[8] K.G. Shojania, A. Jennings, A. Mayhew, et al., The effects of on-screen, point of care computer reminders on processes and outcomes of care, Cochrane Database Syst. Rev. 3 (2009), CD001096.

[9] K.G. Shojania, A. Jennings, A. Mayhew, et al., Effect of point-of-care computer reminders on physician behaviour: a systematic review, CMAJ 182 (2010) E216-E225.

[10] A.J. Long, P. Chang, The effect of using the health smart card vs. CPOE reminder system on the prescribing practices of non-obstetric physicians during outpatient visits for pregnant women in Taiwan, Int. J. Med. Inform. 81 (2012) 605-611.

[11] S. Eslami, N.F. de Keizer, D.A. Dongelmans, et al., Effects of two different levels of computerized decision support on blood glucose regulation in critically ill patients, Int. J. Med. Inform. 81 (2012) 53-60.

[12] S. Herzberg, K. Rahbar, L. Stegger, et al., Concept and implementation of a computer-based reminder system to increase completeness in clinical documentation, Int. J. Med. Inform. 80 (2011) 351-358.

[13] J.O. Zaat, J.T. van Eijk, H.A. Bonte, Laboratory test form design influences test ordering by general practitioners in The Netherlands, Med. Care 30 (1992) 189-198.

[14] N.R. Kahan, D.A. Waitman, D.A. Vardy, Curtailing laboratory test ordering in a managed care setting through redesign of a computerized order form, Am. J. Manag. Care 15 (2009) 173-176.

[15] V. Shalev, G. Chodick, A.D. Heymann, Format change of a laboratory test order form affects physician behavior, Int. J. Med. Inform. 78 (2009) 639-644.

[16] R. Tervo-Pellikka, The community social and health care system in Finland, Int. J. Biomed. Comput. 39 (1995) 181-186.

[17] Clinical Laboratory Diagnostics, Use and Assessment of Clinical Laboratory Results, in: L. Thomas (Ed.), Ist English ed., TH-Books Verlagsgesellschaft mbH, Frankfurt/Main, Germany, 1998.

[18] R. Bindels, A. Hasman, J.W. van Wersch, et al., Evaluation of an automated test ordering and feedback system for general practitioners in daily practice, Int. J. Med. Inform. 73 (2004) 705-712.

[19] S.R. Ranji, M.A. Steinman, K.G. Shojania, et al., Interventions to reduce unnecessary antibiotic prescribing: a systematic review and quantitative analysis, Med. Care 46 (2008) 847-862.

[20] A. Akbari, A. Mayhew, M.A. Al-Alawi, et al., Interventions to improve outpatient referrals from primary care to secondary care, Cochrane Database Syst. Rev. 4 (2008), CD005471.

[21] L. Forsetlund, A. Bjorndal, A. Rashidian, et al., Continuing education meetings and workshops: effects on professional practice and health care outcomes, Cochrane Database of Syst. Rev. 2 (2009), CD003030.

[22] G. Jamtvedt, J.M. Young, D.T. Kristoffersen, et al., Audit and feedback: effects on professional practice and health care outcomes, Cochrane Database Syst. Rev. 2 (2006), CD000259.

[23] S. Miyakis, G. Karamanof, M. Liontos, et al., Factors contributing to inappropriate ordering of tests in an academic medical department and the effect of an educational feedback strategy, Postgrad. Med. J. 82 (2006) $823-829$.

[24] D. Barefird, A. Hayling, Inappropriate use of laboratory services: long term combined approach to modify request patterns, BMJ 301 (1990) 1305-1307.

[25] P. Axt-Adam, J.C. Van der Wouden, E. van der Does, Influencing behavior of physicians ordering laboratory tests: a literature study, Med. Care 31 (1993) 784-794.

[26] V.H. Verstappen, G. ter Riet, W.I. Dubois, et al., Variation in test ordering behaviour of GPs: professional or context-related factors? Fam. Pract. 21 (2004) 387-395.

[27] I. Bayoumi, M. Al Balas, S.M. Handler, et al., The effectiveness of computerized drug-lab alerts: a systematic review and meta-analysis, Int. J. Med. Inform. 83 (2014) 406-415.

[28] T. Ingebrigtsen, A. Georgiou, R. Clay-Williams, et al., The impact of clinical leadership on health information technology adoption: systematic review, Int. J. Med. Inform. 83 (2014) 393-405.

[29] I. Scott, What are the most effective strategies for improving quality and safety of health care? Intern. Med. J. 39 (2009) 389-400

[30] P.S. Roshanov, J.J. You, J. Dhaliwal, et al., Can computerized clinical decision support systems improve practitioners' diagnostic test ordering behavior? A decision-maker-researcher partnership systematic review, Implement. Sci. 6 (2011) 88 .

[31] M.P. Gagnon, F. Legare, M. Labrecque, et al., Interventions for promoting information and communication technologies adoption in healthcare professionals, Cochrane Database Syst. Rev. 1 (2009), CD006093. 